Reseña

\title{
Paleontología de invertebrados
}

de Ma. Luisa Martínez-Chacón y Pascual Rivas (eds.) 2009, Sociedad Española de Paleontología, Instituto Geológico y Minero de España, Universidad de Oviedo, Universidad de Granada, 524 p.

\author{
por Francisco Sour-Tovar ${ }^{1}$ \\ ${ }^{1}$ Departamento de Biología Evolutiva, Facultad de Ciencias, UNAM, Ciudad Universitaria, México D. F. MEXICO 04510 \\ *fst@hp.fciencias.unam.mx
}

Dada la escasa existencia de libros recientes publicados en español sobre invertebrados fósiles, presento con gusto la reseña de una obra que cubre ese vacío. Para lograr esta obra, 32 especialistas con una amplia experiencia en diferentes grupos biológicos han escrito ocho capítulos en que se consideran los recientes métodos de análisis de invertebrados fósiles, los avances que se han logrado en el estudio de su paleobiología y en los sistemas de clasificación de los organismos fósiles. El primer capítulo desglosa la información más importante sobre el origen y diversificación de los animales, y en los siguientes siete se describe la morfología, anatomía, taxonomía, bioestratigrafía, paleoecología, paleobiogeográfia y la filogenia de poríferos y cnidarios, artrópodos, moluscos, braquiópodos, briozoarios, equinodermos y graptolitos, grupos de invertebrados más abundantes y comunes en el registro fósil. Además, cada capítulo se complementa con abundantes ilustraciones, de gran calidad y sumamente didácticas, con una bibliografía muy bien seleccionada.

El capítulo introductorio consiste de una descripción del registro fósil que abarca desde las primeras etapas de la historia de la vida hasta el surgimiento de la diversidad de invertebrados que caracteriza a la llamada Explosión Cámbrica. Los fósiles más antiguos que se han encontrado y la serie de registros que se tienen a lo largo del Proterozoico y hasta inicios del Cámbrico son analizados dentro de un contexto cronológico, que permite comprender cómo la vida da sus primeros pasos evolutivos seguidos de un proceso que comprende cerca de 3000 millones de años y en el que resalta el surgimiento de las primeras células procariotas, de los primeros seres eucariotas, el desarrollo de la pluricelularidad, la diferenciación tisular, la aparición de partes esqueléticas y la primera gran radiación de los animales. Para detallar esta historia, las biotas fósiles y los yacimientos más importantes que se conocen alrededor de

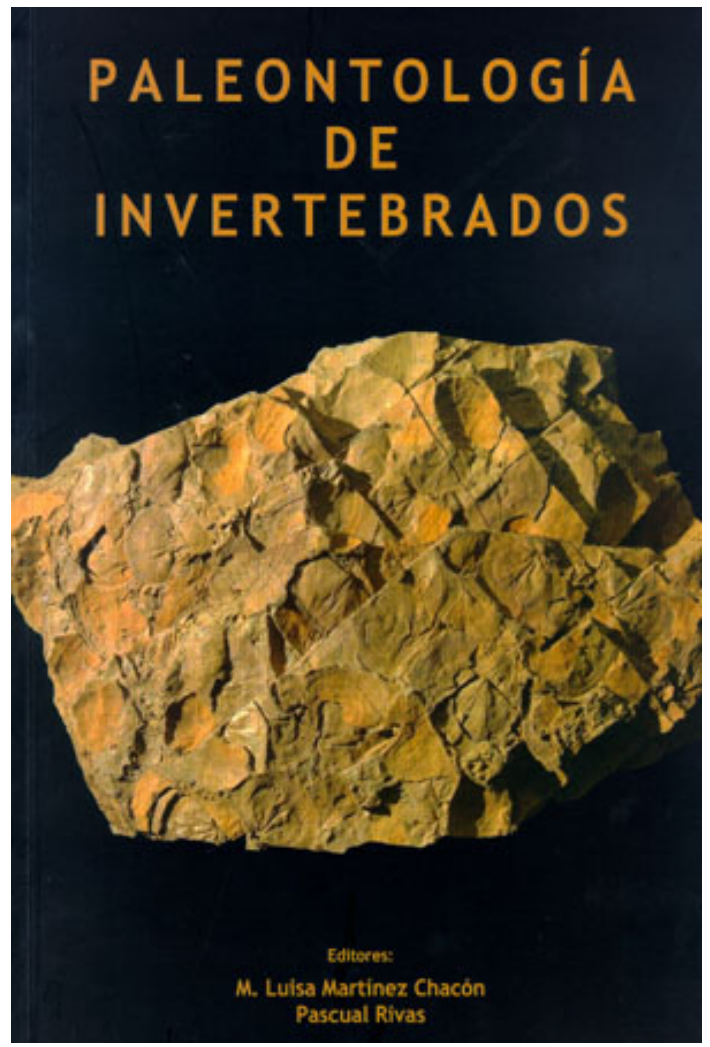

la Tierra, como Ediacara o Burgess Shale, son descritos de manera concisa pero bien ilustrada. También se incluye una bien documentada discusión sobre la importancia de cada uno de los eventos evolutivos mencionados en el origen de la diversidad animal.

El capítulo dos corresponde al tratado de Esponjas y Cnidarios, grupos que, aun cuando carecen entre ellos 
de afinidad filogenética y presentan planes estructurales diferentes, comparten una serie de adaptaciones a hábitos de vida similares. Para la descripción de las esponjas y de cada uno de los grupos que han sido asignados al fílum, se utiliza la clasificación mas reciente que se ha propuesto y que es aceptada por la mayoría de los taxónomos del grupo. Cada grupo es descrito detalladamente y con ilustraciones de gran calidad didáctica, que facilitan la comprensión de los rasgos morfológicos que caracterizan a las clases y órdenes. En el capítulo se resalta el análisis de la distribución temporal y geográfica y de la paleoecología de arqueociátidos, quetétidos y estromatoporoideos, esponjas que llegaron a formar arrecifes en diferentes tiempos geológicos desarrollando morfologías adaptativas muy particulares; este análisis permite comprender la evolución temprana de las comunidades arrecifales y se complementa con la información que se detalla en el apartado correspondiente a corales, que reciben un tratamiento similar al de las esponjas, al detallarse su morfología esquelética, su distribución temporal, sus hábitos y una concisa descripción de la evolución del grupo y la historia geológica de los arrecifes coralinos como bioconstrucciones.

El capítulo tres está dedicado a artrópodos fósiles, entre los que marellomorfos, aracnomorfos, crustáceos, insectos y miriápodos (grupos que por su abundancia y diversidad son los más importantes en el registro fósil) son descritos e ilustrados detalladamente. Puntos sobresalientes en este capítulo son el análisis de la morfología funcional de los trilobites, la discusión sobre la historia geológica de los ostrácodos y un análisis de diversos aspectos tafonómicos e icnológicos asociados al registro fósil de los insectos, que brindan importante información paleoambiental.

El fílum Mollusca es tratado en el capítulo cuatro, el más extenso de la obra y en el que sus autores, siguiendo el plan general del libro, describen con gran detalle las características del grupo. En particular, bivalvos, gasterópodos y cefalópodos son tratados e ilustrados con gran profundidad. También son analizados brevemente otros moluscos menos abundantes en el registro fósil, entre ellos monoplacóforos, bellerofontiformes, rostroconchos, escafópodos, poliplacóforos, tentaculitoidos y hiolitidos; para cada uno de ellos se describe su importancia como fósiles de interés filogenético, bioestratigráfico o paleoambiental.

En el capítulo del fílum Brachiopoda, cuyos representantes son sumamente abundantes en el registro fósil (sobre todo en el Paleozoico), la morfología se describe con gran detalle y desde una perspectiva funcional y adaptativa. Esto enriquece la lectura y permite comprender el porqué de la gran variabilidad que se presenta en todos y cada uno de los rasgos que caracterizan a este tipo de lofoforados. Este capítulo es la primera obra en español y de las primeras en cualquier otro idioma, que utilizan la muy reciente clasificación propuesta para el grupo y que es fruto de la colaboración de un extenso grupo de especialistas que, a lo largo de más de 15 años, han trabajado en la revisión de todos los taxa supraespecíficos de braquiópodos descritos para el registro fósil. Con ello, resalta la utilidad de la obra que se reseña como referencia bibliográfica y facilita a cualquier interesado en realizar un trabajo sistemático sobre el grupo. El mismo capítulo presenta una descripción de los modos de vida de braquiópodos, su relación con el sustrato, aspectos sobre su ecología y paleoecología y resume claramente cuál es la importancia de los braquiópodos fósiles como indicadores paleoambientales, bioestratigráficos y en la reconstrucción de paleogeografías.

El capítulo seis mantiene el carácter general de la obra, describiendo e ilustrando con detalle la morfología, anatomía, filogenia y sistemática de los diferentes grupos de briozoarios y se analizan sus hábitos reproductivos, patrones de desarrollo y los tipos y la morfología funcional de colonias que forman. Con ello se hace comprensible cómo, con el análisis de la morfología colonial, se pueden inferir batimetrías, aspectos sedimentológicos y otros rasgos paleoambientales, y también se resalta el papel de los briozoarios como productores de sedimentos que llegan a formar grandes depósitos que caracterizan diversas facies sedimentarias. La sección en que se trata la taxonomía permite comprender fácilmente la clasificación a nivel de órdenes y posee ilustraciones, como en el resto de los capítulos del libro, bien seleccionadas y de gran calidad.

La sección dedicada a equinodermos también representa una referencia de gran utilidad, sobre todo a quien se inicia en el estudio del grupo, donde se detalla el plan estructural del fílum y sus variaciones dentro de las diferentes clases que se han reconocido. Se analizan las relaciones filogenéticas entre los sub-grupos y sobresalen las descripciones de la diversidad de clases de equinodermos que limitaron su existencia al Paleozoico. Las formas post-paleozoicas también se tratan con detalle y se resalta su paleobiología y su evolución.

El último capítulo del libro está dedicado a los graptolitos, grupo de hemicordados coloniales que se asigna al Subfílum Pterobranchia y cuya importancia, sobre todo bioestratigráfica, los hacen merecedores de ser incluidos en este tratado. Por ello se describe a detalle su morfología individual y colonial, se analizan las características de las colonias como su estructura y composición, y las afinidades que su esqueleto presenta con otros grupos de hemicordados. El capítulo también incluye un análisis muy claro sobre la clasificación del grupo, su evolución, paleoecología y paleobiogeografía.

En resumen, la Paleontología de Invertebrados que se reseña representa una obra de consulta de enorme utilidad, no sólo para estudiantes que se enfrentan por primera vez al estudio de un invertebrado fósil, también para todos los paleontólogos que nos hemos especializado en el estudio de un grupo de organismos en particular y quienes encontraremos en esta obra la información actualizada sobre los grupos de invertebrados fósiles más abundantes y comunes del registro fósil. 